\title{
EFICACIA DE LA TERAPIA DE GRUPO EN CÁNCER DE MAMA: EVO- LUCIÓN DE LAS EMOCIONES DESADAPTATIVAS
}

\author{
EFFECTIVENESS OF A GROUP THERAPY IN BREAST CANCER: DEVELOPMENT OF \\ MALADAPTIVE EMOTIONS
}

Eva Rodríguez y Antoni Font

Universitat Autònoma de Barcelona

Resumen

El estado emocional de las mujeres con cáncer de mama puede variar a lo largo del tiempo, en función de las dificultades físicas y psicosociales que se pueden encontrar a lo largo de la enfermedad, y que pueden continuar una vez finalizado el proceso de los tratamientos oncológicos. Cuando la paciente espera volver a recuperar su vida cotidiana, con frecuencia se mantienen emociones negativas como el miedo a las recaídas o sentirse deprimido. El objetivo del presente estudio longitudinal es conocer como evolucionan estas emociones desadaptativas, cuando se participa en una terapia de grupo orientada a mejorar la calidad de vida. La muestra final se compone de $\mathbf{3 8}$ mujeres con cáncer de mama que se encuentran en intervalo libre de enfermedad. Los resultados obtenidos indican que el denominado "malestar emocional" mejora a lo largo de la terapia, si bien en algunas pacientes afloran más las emociones negativas durante la terapia que al inicio de la misma. Donde se observa una mayor mejoría es en el aspecto de recuperar las ganas e ilusión por las cosas $(p<0,01)$. También observamos una diminución de la ira, la ansiedad, la depresión, las dificultades de concentración y el miedo. Concluimos que la terapia de grupo de larga duración (8 meses) utilizada, se ha mostrado eficaz para ayudar a la adaptación emocional.

Palabras clave: Cáncer de mama, terapia de grupo, eficacia, evolución emocional, adaptación.

\section{Abstract}

The emotional state of women with breast cancer may vary over time, depending on the physical and psychosocial difficulties that can be found throughout the disease, and that may continue once the process of cancer treatments finished. When the patient expects to recover his everyday life, negative emotions such as fear of relapses or feeling depressed, often remain. The objective of this longitudinal study is to know how these maladaptive emotions progressed, when participating in group therapy aimed at improving the quality of life. The final sample consists of 38 women with breast cancer in disease-free interval. The results indicate that the so-called "emotional distress" improves over therapy, although by many patients negative emotions emerge more during the therapy than at the beginning. There is a greater improvement in the aspect of recovering the motivation and enthusiasm for doing something $(p<0,01)$. We also observed a diminution of anger, anxiety, depression, concentration difficulties and fear. We conclude that the long-term (8 months) therapy has been shown effective to help emotional adjustment.

Key words: Breast cancer, group psychotherapy, efficacy, emotional evolution, adaptation.

\section{Correspondencia:}

Antoni Font

Universidad Autónoma de Barcelona. Facultad de Psicología. Seminario de Psicología Oncológica

08193 Cerdanyola del Vallés (Bellaterra). Barcelona

E-mail: Antonio.font@uab.cat 


\section{INTRODUCCIÓN}

El cáncer de mama es uno de los problemas de salud más importantes, tanto por sus efectos como por su frecuencia. En la Unión Europea, se diagnostican aproximadamente 450.000 casos nuevos y la mortalidad se sitúa en torno a los 139.000 casos (1). En España, el cáncer de mama es el tumor más frecuente en mujeres, se diagnostican aproximadamente 22.000 casos nuevos al año (casi el 30\% de todos los tumores del sexo femenino en nuestro país), y mueren unas 6.295 mujeres anualmente $(16,7 \%)$ con una media de edad de 66 años. Los programas de detección precoz junto con los avances diagnósticos y terapéuticos se han traducido en un incremento de la supervivencia a los 5 años, que en España se sitúa ya alrededor del $82 \%{ }^{(2-4)}$.

El diagnóstico y el tratamiento del cáncer de mama comporta muchas y variadas reacciones emocionales, que pueden ser de shock, incredulidad, miedo, ansiedad, depresión, sensación de pérdida de control, disminución de la autoestima, cambios en la imagen corporal, etc.; que pueden oscilar y mantenerse a lo largo del tiempo, disminuyendo la calidad de vida de las personas afectadas ${ }^{(5-8)}$. Así pues, los efectos de la enfermedad no siempre finalizan cuando se acaban los tratamientos oncológicos, siendo más frecuente que diversas dificultades físicas y emocionales persistan en el tiempo. Por ejemplo, Lemieux et al ${ }^{(9)}$, indican que más del $40 \%$ de los pacientes presentan síntomas clínicamente relevantes de malestar psicológico en las fases de seguimiento. Posiblemente la disminución en el funcionamiento familiar y social, la disminución en la participación de actividades lúdicas y la afectación en la sexualidad, son las consecuencias negativas más significativas a largo plazo del diagnóstico de cáncer. En un estudio realizado por Bleiker et al. ${ }^{(10)}$ con 244 mujeres con cáncer de mama, se observó que entre el $20-30 \%$ de las pacientes presentaban malestar severo durante el primer año después de la cirugía, y a los dos años, una quinta parte de las mujeres presentaban todavía altos niveles de pensamientos intrusivos respecto a la enfermedad.

Es conocido que el cáncer de mama es una enfermedad crónica cuya evolución resulta en gran parte impredecible. Esta incertidumbre continua puede explicar el alto índice de morbilidad psicológica que se mantiene durante tiempo después del diagnóstico inicial. Diversos autores coinciden en señalar que las dificultades emocionales más frecuentes son: miedo a la recidiva y/o a la muerte, sensación de pérdida de control, aislamiento, problemas de adaptación a los cambios físicos (pérdida de energía, cansancio, infertilidad, ganancia de peso, cambios en los patrones de sueño y alimentación, etc.), malestar psicosocial (ansiedad, depresión, baja autoestima y cambios en la imagen corporal), dificultades en las relaciones sexuales, redefinición o cambio de valores, cambios en las relaciones con las personas del entorno (familiares y amigos) y preocupaciones relacionadas con la actividad laboral| ${ }^{(11-14)}$.

En un estudio realizado por Hodgkinson et al. ${ }^{(15)}$ con 117 mujeres supervivientes de cáncer de mama con tiempos desde el diagnóstico entre 2-10 años, los autores encontraron que las pacientes manifestaron ansiedad y necesidades de apoyo (86\%), principalmente relacionadas con cuestiones existenciales como tratar las preocupaciones sobre la recidiva del cáncer (manifestada por una tercera parte de las supervivientes) y necesidades informativas. Resultados que también se confirman en el estudio de Rosedale y Krainovich-Miller ${ }^{(16)}$ realizado también con mujeres supervivientes, los autores encontraron que las pacientes que no se sienten informadas sobre las posibles secuelas físicas y emocionales de la supervivencia, presentan niveles más elevados de malestar emocional e incertidumbre a lo largo del tiempo. Todos 
estos datos nos sugieren que las mujeres con cáncer de mama supervivientes presentan una serie de necesidades que, en ocasiones, independientemente del tiempo transcurrido desde el diagnóstico de la enfermedad, no están cubiertas.

Ante ello, se han propuesto diferentes formas de apoyo psicológico y terapias que pueden mejorar la calidad de vida de los pacientes oncológicos y facilitar el proceso de adaptación a la enfermedad (17-20). Uno de los abordajes terapéuticos cuyos efectos beneficiosos han sido contrastados en los últimos años, también en nuestro país ${ }^{(21-24)}$ es la terapia de grupo. Este tipo de intervención facilita el cambio de actitudes, conductas y emociones, a través de la reflexión conjunta, el intercambio de experiencias, el autoconocimiento y el apoyo mutuo ${ }^{(25-27)}$. La mayoría de trabajos realizados hasta el momento son intervenciones cortas, entre 6 y 10 sesiones semanales, e incluso se han realizado intervenciones grupales de fin de semana, que trabajan diferentes temas relacionados con la enfermedad, como la ansiedad, los miedos, la comunicación con el entorno, la autoestima, etc. ${ }^{(28-31)}$.

El objetivo del presente estudio es conocer la evolución emocional que siguen las pacientes con cáncer de mama, participantes en una intervención grupal de larga duración, concretamente a lo largo de 8 meses (20 sesiones), a fin de observar la posible eficacia de la terapia en la mejora de las emociones negativas o desadaptativas.

\section{MÉTODO}

\section{Participantes}

Se contactó con 172 pacientes con cáncer de mama que solicitaban apoyo psicológico en grupo en la Asociación Española contra el Cáncer de Barcelona. Los datos se recogieron mediante consentimiento informado por escrito de todas las participantes, y asegurando el anonimato de los datos cuya evaluación se realizará únicamente a nivel grupal.

Los criterios de inclusión al grupo de apoyo fueron: Mujer con cáncer de mama con dificultades psicosociales debido al diagnóstico, la enfermedad o su tratamiento como, por ejemplo, ansiedad, depresión, estrés, tristeza, agotamiento emocional, falta de ilusión, etc; y paciente que necesite o desee comunicar y compartir su experiencia con personas que han sufrido la misma enfermedad. Criterios de exclusión: Paciente con cáncer de mama con metástasis a distancia; estar en fase de tratamiento activo (excepto tratamiento hormonal adyuvante) encontrarse en fase de recidiva; presencia de dos tumores primarios diferentes; presencia de otras enfermedades importantes asociadas que pueden interferir en el seguimiento de la terapia; presencia de trastorno psiquiátrico importante e imposibilidad de comunicación verbal.

Aplicados estos criterios, la muestra disponible para el presente estudio se componía inicialmente de 96 mujeres. Al tratarse de una terapia de larga duración, algunas pacientes abandonaron la terapia antes de su finalización (33 casos), siendo el motivo más frecuente el encontrase mejor y no necesitar más sesiones de terapia. Los análisis de evolución emocional que aquí presentamos se refieren a aquellas pacientes que han asistido de forma regular a la terapia de grupo hasta el final y que además realizaron las tres valoraciones de la calidad de vida, es decir, 38 pacientes (se excluyen por tanto los datos de las pacientes que pese a finalizar la terapia, no estuvieron presentes en una de las valoraciones).

\section{Instrumentos}

1) Datos sociodemográficos e historia clínica

Los datos personales incluían edad, estado civil, nivel de estudios y situación laboral. El historial clínico recogía datos 
médicos oncológicos (diagnóstico, fase de la enfermedad en que se encontraba cada paciente y los tratamientos médicos recibidos), antecedentes familiares, datos de la historia psicológica (antecedentes psicopatológicos personales, resultados de la evaluación inicial -nivel sintomático, nivel conductual, nivel cognitivo, apoyo sociofamiliar y problemas socioeconómicos-), objetivos terapéuticos, actuación psicológica y motivo de alta.

2) Sub-escala emocional del Cuestionario de calidad de vida QL-CA-Afex

La valoración de la sintomatología emocional se ha basado en el instrumento desarrollado por Font ${ }^{(32,33)}$ y Font y Bayés ${ }^{(34)}$ para pacientes de cáncer, para ser administrado principalmente en pacientes en régimen ambulatorio, que valora la calidad de vida en relación con el estado de salud. Consta de 27 ítems (escalas análogo-visuales) que se puntúan de $0 \mathrm{a}$ 100 , y que se agrupan en torno a cuatro subescalas (síntomas, dificultades con los hábitos cotidianos, dificultades familiares y sociales y malestar emocional). El último ítem del cuestionario se considera una escala global de la calidad de vida. Este instrumento se ha desarrollado con población española y presenta una validez y fiabilidad satisfactorias, permite detectar las variaciones en calidad de vida a lo largo de la enfermedad ${ }^{(33-36)}$. Se ha utilizado con pacientes oncológicos en diferentes fases de la enfermedad ${ }^{(37-41)}$.

El estado emocional de las pacientes se valoró sucesivamente, con las 7 escalas análogo-visuales del instrumento (ansiedad, miedo, depresión, ira, pérdida de ilusión, dificultades de concentración, e insatisfacción con el aspecto físico). Una mayor puntuación en un ítem significa más malestar emocional. El análisis de fiabilidad realizado con las 96 pacientes que inician la terapia de grupo arrojó un valor Alfa de Crombach de 0,816, por lo que la escala emocional empleada se pudo considerar suficientemente fiable.

\section{Procedimiento}

En una primera entrevista individual, realizada por una psicooncóloga, se recogían los datos de la historia clínica y psicológica, se valoraba el estado emocional de la paciente, y posteriormente, se procedía a la inclusión de la paciente en un grupo de apoyo, en función del cumplimiento o no de los criterios clínicos y psicológicos anteriormente indicados. En el caso de que no se incluyera la paciente en un grupo, se le ofrecía la posibilidad de recibir apoyo psicológico individual. Para conocer la evolución emocional de las pacientes a lo largo de los 8 meses de intervención grupal, se realizaron tres valoraciones: la primera, antes de iniciar la terapia; la segunda valoración se realizó transcurridos 4 meses $\left(11^{\text {a }}\right.$ sesión, es decir, hacia la mitad de la terapia), y la última valoración, al finalizar la intervención de grupo.

\section{Intervención psicológica en grupo}

La intervención consistía en una terapia de apoyo psicológico en grupo monitorizado por una profesional de la psicología. Cada grupo estaba formado por un mínimo de 5 y un máximo de 10 personas. Las reuniones se realizaban quincenalmente, durante una hora y media, con una duración aproximada de 8 meses, es decir, 20 sesiones por grupo.

La terapia de grupo aplicada combina la expresión de emociones (terapia emotivo-expresiva) con la práctica de técnicas cognitivo-conductuales para reducir las dificultades emocionales derivadas del diagnóstico y tratamiento de la enfermedad, facilitar la adaptación y mejorar la calidad de vida. Al inicio de cada sesión, se permite un tiempo y un espacio para que la paciente pueda comunicar cómo 
se ha sentido el período entre sesiones, incidiendo en las vivencias emocionales de los acontecimientos diarios. Este espacio se permite especialmente en las primeras 3 sesiones donde las pacientes se van conociendo y se van creando los primeros vínculos de apoyo entre las participantes. A partir de la $4^{\text {a }}$ sesión y a lo largo de la terapia se van trabajando diferentes temas psicológicos que se pueden englobar en cuatro módulos: ansiedad, depresión, autoestima e imagen corporal, relaciones afectivas y sexualidad. Cada módulo consta de tres sesiones de trabajo, donde se expone una parte teórica del tema en cuestión, se identifican las estrategias utilizadas por las pacientes para afrontar cada dificultad emocional en particular $y$, a continuación, se enseñan una serie de estrategias o técnicas psicológicas para su afrontamiento más adaptativo.

\section{Análisis estadístico}

El análisis estadístico ha sido realizado por el Servicio de Estadística de la Universidad Autónoma de Barcelona (programa SPSS, versión 14.0 para Windows). Para la comparación del estado emocional de las pacientes se ha utilizado el test de Friedman para datos apareados.

\section{RESULTADOS}

\section{Características de la muestra}

Las características sociodemográficas y clínicas de la muestra se describen en la tabla 1. La muestra final está formada por 38 mujeres de las 96 que participaron en la terapia, es decir, aquellas de las que se disponía de las tres valoraciones a lo largo de los 8 meses. La edad media es de 48 años, y desviación típica de 7,1, con edades comprendidas entre 35 y 65 años. La mayor parte de las mujeres están casadas $(84,2 \%)$, tienen hijos $(86,8 \%)$ y más de la mitad de las pacientes tienen estudios primarios $(63,2 \%)$. Respecto a los datos clínicos, tal como ya se ha indicado, todas presentan cáncer de mama no metastásico. A una proporción importante de mujeres se les ha practicado una mastectomía (65,8\%); más de la mitad de las pacientes han recibido quimioterapia $(65,8 \%)$, la mitad ha realizado radioterapia $(50 \%)$ y la mayoría siguen tratamiento hormonal (76,3\%). Respecto a la fase de la enfermedad en el momento de la entrevista, la mayoría se encuentran en fase de intervalo libre de la enfermedad (71\%), y el resto, en fase de primer tratamiento (29\%). Al finalizar la terapia de grupo, todas ellas han finalizado los tratamientos oncológicos, exceptuando el tratamiento hormonal y se encuentran por tanto en fase de intervalo libre de enfermedad (100\%).

Comparación de las emociones desadaptativas antes, durante y después de la terapia de grupo

La prueba de comparaciones para datos apareados indica que la subescala de malestar emocional presenta una diferencia importante entre medianas. Es decir, al finalizar la intervención, las pacientes evolucionan emocionalmente de forma favorable $(\mathrm{P}<0,02)$, teniendo en cuenta su estado de salud, que cuando iniciaron la terapia (tabla 2).

Todos los aspectos concretos que componen el malestar emocional evolucionaron favorablemente a lo largo de la terapia (figura 1).

Los resultados muestran que la "pérdida de ilusión" mejora significativamente a lo largo del tiempo $(P<0,01)$. Asimismo la "ira", la "ansiedad", la "depresión", las "dificultades de concentración" y el "mie$\mathrm{do}^{\prime \prime}$, van mejorando a lo largo de la terapia de grupo. El ítem "insatisfacción con el aspecto físico" mejora durante la terapia, para empeorar ligeramente, al finalizar la misma. La ansiedad, la depresión y el miedo son los tres ítems con las puntuaciones 


\section{Tabla 1. Características sociodemográficas y clínicas de la muestra}

\begin{tabular}{|c|c|c|}
\hline \multicolumn{3}{|l|}{ Edad } \\
\hline & Media, desviación típica & $48 ; 7,1$ \\
\hline & Rango & $35-65$ \\
\hline \multicolumn{3}{|c|}{ Estado civil } \\
\hline & Soltera & $1(2,6 \%)$ \\
\hline & Casada & $32(84,2 \%)$ \\
\hline & Divorciada & $2(5,3 \%)$ \\
\hline & Viuda & $2(5,3 \%)$ \\
\hline & Pareja estable & $1(2,6 \%)$ \\
\hline \multicolumn{3}{|l|}{ Hijos } \\
\hline & $\mathrm{Si}$ & $33(86,8 \%)$ \\
\hline & No & $5(13,2 \%)$ \\
\hline \multicolumn{3}{|c|}{ Estudios } \\
\hline & Primarios & $24(63,2 \%)$ \\
\hline & Medios & $7(18,4 \%)$ \\
\hline & Superiores & $7(18,4 \%)$ \\
\hline \multicolumn{3}{|c|}{ Tratamientos oncológicos } \\
\hline & Cirugía conservadora & $13(34,2 \%)$ \\
\hline & Mastectomía & $25(65,8 \%)$ \\
\hline & Quimioterapia & $25(65,8 \%)$ \\
\hline & Radioterapia & $19(50 \%)$ \\
\hline & Hormonoterapia & $29(76,3 \%)$ \\
\hline & Braquiterapia & $2(5,3 \%)$ \\
\hline \multicolumn{3}{|c|}{ Fase de la enfermedad ( $1^{\text {a }}$ valoración) } \\
\hline & Primer tratamiento & $11(29 \%)$ \\
\hline & Intervalo libre & $27(71 \%)$ \\
\hline \multicolumn{3}{|c|}{ Fase de la enfermedad ( $2^{a}$ valoración) } \\
\hline & Intervalo libre & $27(71 \%)$ \\
\hline
\end{tabular}

Tabla 2. Evolución global del malestar emocional ( $\mathbf{N}=\mathbf{3 8}$ pacientes)

\begin{tabular}{|c|c|c|c|}
\hline Momento de la evaluación & Media & Mediana & Desviación tipo \\
\hline Antes de la terapia de grupo & 31,7 & 29,6 & 17,1 \\
\hline Durante la terapia & 28,1 & 26,8 & 16,5 \\
\hline Después de la terapia & 26,2 & 24,9 & 17,7 \\
\hline
\end{tabular}


Figura 1. Valores medios de los aspectos emocionales negativos antes, durante $y$ después de la terapia de grupo de larga duración ( 8 meses) ( $N=38$ pacientes)

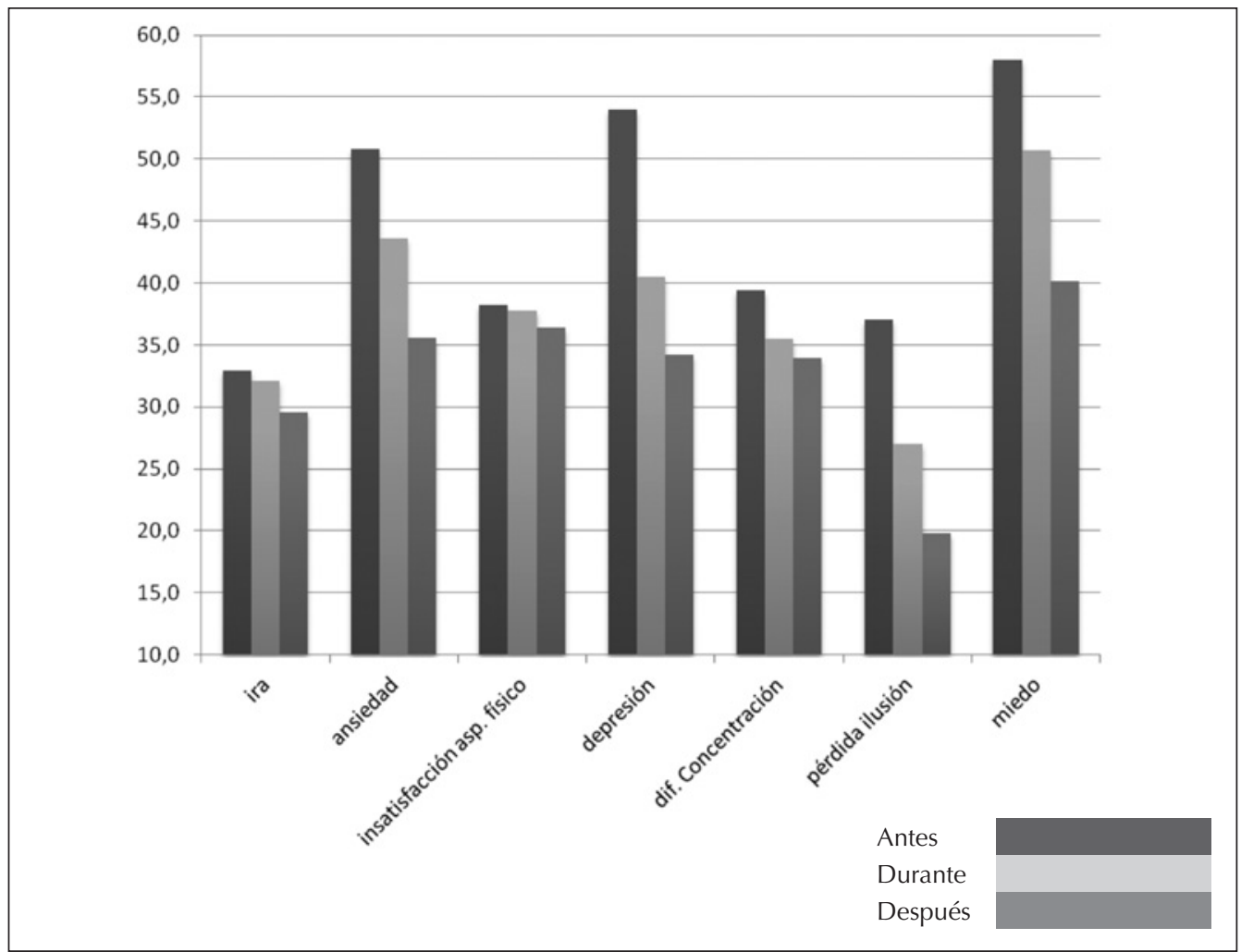

Tabla 3. Comparación de estados emocionales antes, durante y después de la intervención grupal

\begin{tabular}{|l|l|c|c|c|c|c|}
\hline \multicolumn{2}{|c|}{$\begin{array}{c}\text { Componentes del } \\
\text { MALESTAR EMOCIONAL }\end{array}$} & \multicolumn{3}{c|}{ Momento evaluación } & \multirow{2}{*}{ FRIEDMAN } & \multirow{2}{*}{ SIG } \\
\cline { 3 - 5 } & Antes & Durante & Después & \\
\hline Ira & Mediana & 30 & 22 & 19 & 1,67 & 2,14 \\
\hline Ansiedad & Mediana & 53 & 45 & 25 & 2,43 & 0,3 \\
\hline $\begin{array}{l}\text { Insatisfacción con } \\
\text { aspecto físico }\end{array}$ & Mediana & 44 & 27 & 32 & 1,63 & 0,44 \\
\hline Depresión & Mediana & 60 & 38 & 29 & 2,1 & 0,35 \\
\hline $\begin{array}{l}\text { Dificultades de } \\
\text { Concentración }\end{array}$ & Mediana & 42 & 37 & 25 & 1,24 & 0,54 \\
\hline Pérdida ilusión & Mediana & 32 & 24 & 10 & $\mathbf{1 0 , 3 8}$ & $\mathbf{0 , 0 0 6}$ \\
\hline Miedo & Mediana & 55 & 47 & 32 & 4,54 & 0,1 \\
\hline
\end{tabular}




\section{Tabla 4. Evolución de la calidad de vida global ( $N=38$ pacientes)}

\begin{tabular}{|c|c|c|c|}
\hline Momento de la evaluación & Media & Mediana & Desviación tipo \\
\hline Antes de la terapia de grupo & 49,3 & 48 & 29,5 \\
\hline Durante la terapia & 35,1 & 30 & 24,3 \\
\hline Después de la terapia & 26,4 & 21 & 23,3 \\
\hline
\end{tabular}

Valor Friedman: 6,44 $(P=0,04)$

más elevadas de esta subescala (ver tabla 3). Por otra parte, la valoración global de la calidad de vida, también evolucionó favorablemente, de manera significativa, a lo largo de la terapia (tabla 4).

\section{DISCUSIÓN}

En el análisis de la evolución de las emociones a lo largo de la terapia de grupo, se pudo observar que las pacientes mejoraban en todas las emociones y en la puntuación global de malestar emocional, en comparación con la situación antes de la terapia. Es decir, al finalizar la intervención, las mujeres participantes presentan menor malestar emocional, que cuando iniciaron el grupo de apoyo. También se observa una recuperación de la satisfacción con el aspecto físico, aunque no llega a ser significativa. En cambio, si que se muestran estadísticamente significativas la mejora del estado emocional conjunto, la recuperación de la ilusión por las cosas y las actividades y la valoración global de la calidad de vida.

En este estudio, los ítems con las puntuaciones más elevadas son el miedo, la depresión y la ansiedad, síntomas que suelen estar muy relacionados, y como se ha podido constatar en estudios anteriores, son los que crean mayor malestar psicológico y, por tanto, más disminuyen la calidad de vida ${ }^{(37,42-44)}$. También son los aspectos que más mejoran, pero continúan estando presentes al finalizar la terapia, lo que indica que el apoyo psicológico debe dirigirse a manejar, más que eliminar, este tipo de emociones.

La ansiedad continua siendo uno de los síntomas emocionales más presentes y que puede fluctuar a lo largo del tiempo, en función de las dificultades que tiene que afrontar la paciente con cáncer de mama en su proceso de enfermedad, coincidiendo con los datos observados en otros estudios. Por ejemplo, en el estudio de Schou et al. ${ }^{(45)}$ se observó que el $34 \%$ de las pacientes mostraban ansiedad antes de la cirugía y al año había disminuido al $26 \%$. Además, en la mayoría de estudios, la ansiedad es más frecuente que la depresión, otro aspecto emocional frecuente en este tipo de pacientes. Por ejemplo, Mehnert y $\mathrm{Koch}^{(46)}$ en una muestra de 1083 mujeres, encontraron que el $19 \%$ presentaban ansiedad y el $11 \%$ depresión.

Las mejorías observadas a nivel de disminución de la depresión y la ansiedad y el miedo sugieren que la terapia de grupo puede ser una alternativa a los tratamientos psicofarmacológicos. La terapia se muestra efectiva para recuperar la ilusión por las cosas: El compartir experiencias y nuevas formas de afrontamiento con otras personas en la misma situación, facilita que las pacientes ganen en seguridad en sí mismas, recuperen sus ilusiones anteriores y pierdan el miedo a desarrollar nuevos proyectos. Una mayor aceptación de la enfermedad y de los efectos de los 
tratamientos, suele acompañarse también de una disminución de la ira.

A lo largo de las sesiones de la terapia hemos podido observar que algunas pacientes presentan más problemas emocionales hacia la mitad de la terapia que al inicio de la misma. Puede contribuir a ello la mayor concienciación que se obtiene en la terapia de grupo respecto a qué apoyos podemos y debemos esperar de los que nos rodean. Algunos aspectos pasan a ser más problemáticos inicialmente, pero después mejoran y las pacientes se adaptan a la nueva situación de supervivencia. Actualmente tenemos tendencia a disminuirlo todo, a intentar no sufrir, pero en ocasiones, no damos tiempo a que las personas se adapten. Las emociones necesitan su tiempo de evolución y posiblemente inicialmente tenemos que estar peor, expresar todas las emociones, principalmente las negativas, para que posteriormente, podamos estar mejor. La terapia nos hace ser más conscientes de cómo nos sentimos, pero ello no es necesariamente negativo, al contrario, ventilar las emociones ayuda a disminuir la tensión que provocan y permite elaborarlas de forma que las podamos canalizar de forma adaptativa.

También es importante destacar que muchas mujeres afirman que no consideran su vida más insatisfactoria que antes, sino que su vida actual es diferente, con aspectos que empeoran, pero también con aspectos que mejoran, como el cambio de valores, ganando muchas de ellas en calidad de vida, mejor incluso que la que tenían antes del diagnóstico de la enfermedad. En estos períodos de confusión e incertidumbre, las personas pueden buscar activamente un significado en un intento de entender porque les ha ocurrido. La terapia facilita la reflexión de la propia vida, pudiéndose convertir la enfermedad en un punto de inflexión, a partir de la cual hacer cambios en un sentido positivo ${ }^{(13,47,48)}$.
Con estos resultados podemos concluir que las pacientes presentan una mejoría en la evolución de sus emociones a lo largo de la terapia de grupo, y al mismo tiempo, la intervención en grupo ayuda a mantener o mejorar la calidad de vida de las mujeres con cáncer de mama, especialmente aquellos aspectos que hacen referencia a los síntomas emocionales, después del diagnóstico de la enfermedad. La mejoría observada, no es únicamente producto del paso del tiempo, sino del aprendizaje de estrategias para facilitar la adaptación y aceptación de la enfermedad y sus posibles secuelas ${ }^{(49-53)}$. Por último, podemos distinguir entre aspectos emocionales más variables (miedo, ansiedad, ilusión por las cosas) y más estables (tendencia a la ira, insatisfacción con el aspecto físico, efectos en la concentración).

A nuestro entender, la terapia se ha mostrado muy efectiva para la mayoría de las pacientes ya que todas las emociones negativas han mejorado a lo largo del tiempo, si bien los resultados estadísticos no han sido significativos en todas las emociones consideradas. No obstante, hay que tener en cuenta que hubo muchas pacientes que no asistieron hasta el final de las sesiones (8 meses después), y la principal razón aducida fue la de que ya no lo necesitaban. En otras palabras: las que siguieron hasta el final y componen los resultados de esta investigación, eran, probablemente, las más necesitadas. En todo caso, el relativamente pequeño número de sujetos consideremos es la principal limitación del presente trabajo.

Los resultados de este estudio nos lleva a recomendar la intervención en grupo como una forma de mejorar la calidad de vida de las mujeres supervivientes de un cáncer de mama, y que los profesionales de la oncología deberían recomendar como una forma de atención integral a este tipo de pacientes, mientras que los profesionales psicoterapeutas no pueden descuidar que el 
aprender a manejar las emociones es determinante en el mantenimiento y/o recuperación de la calidad de vida.

AGRADECIMIENTOS: A todas las mujeres con cáncer de mama y a la Asociación Española contra el Cáncer de Barcelona por facilitar el desarrollo de este trabajo.

\section{REFERENCIAS BIBLIOGRÁFICAS}

1. Globocan 2008. Breast cancer incidence, mortality and prevalence worldwide in 2008. Summary. Cancer Fact Sheet. International Agency for Research on cancer (IARC). [Acceso 3 de enero de 2013]. Disponible en: http://globocan.iarc.fr/factsheet.asp

2. Asociación Española contra el Cáncer (AECC). Incidencia del cáncer de mama. [Acceso 15 de noviembre de 2012]. Disponible en: https://www.aecc.es/SobreElCancer/CancerPorLocalizacion/CancerMama/Paginas/incidencia.aspx

3. Asociación Española contra el Cáncer (AECC). Pronóstico (supervivencia y mortalidad) del cáncer de mama. [Acceso 15 de noviembre de 2012]. Disponible en: https://www.aecc.es/SobreElCancer/CancerPorLocalizacion/CancerMama/Paginas/ pronostico.aspx

4. Cabanes A, Pérez-Gómez B, Aragonés N, Pollán M, López-Abente G. La situación del cáncer en España. 1975-2006. Madrid: Centro Nacional de Epidemiología. Instituto de Salud Carlos III, 2009.

5. Avis NE, Crawford S, Manuel J. Quality of life among younger women with breast cancer. J Clin Oncol 2005;23:3322-30. Doi: 10.1200/JCO.2005.05.130

6. Michael Y, Berkman L, Colditz G, Holmes $M$, Kawachi, I. Social networks and healthrelated quality of life in breast cancer survivors. A prospective study. J Psychosom Res 2002;52:285-93.

7. Pugliese P, Fabi A, Maggi G, Falcicchio C, Cognetti F. Impact of surgery and adjuvant treatment on the quality of life of women with breast carcinoma: a prospective study. Trabajo presentado en el 8th World Congress of Psycho-Oncology, Venecia, octubre 2006.

8. Schnoll RA, Knowles JC, Harlow L. Correlates of adjustment among cancer survivors. J Psychosoc Oncol 2002;20:37-59. Doi: 10.1300/J077v20n01_03

9. Lemieux J, Bordeleau L, Goodwin, P. Medical, psychosocial, and health-related quality of life issues in breast cancer survivors. En: Ganz P, editor. Cancer survivorship. Today and tomorrow. New York: Springer, 2007.p.122-44.

10. Bleiker EMA, Pouwer F, Van der Ploeg HM. Psychosocial distress two years after diagnosis of breast cancer: frequency and prediction. Patient Edu Couns 2000;40:209-17.

11. Allen JD, Savadatti S, Levy AG. The transition from breast cancer "patient" to "survivor". Psychooncology 2009;18:71-8. Doi: 10.1002/pon.1380

12. Arndt V, Stegmeier C, Ziegler H, Brenner $\mathrm{H}$. A population-based study of the impact of specific symptoms on quality of life in women with breast cancer 1 year after diagnosis. Cancer 2006;107:2496-503. Doi: 10.1002/cncr.22274

13. Bower JE, Meyerowitz BE, Desmond KA, Bernaards CA, Rowland JH, Ganz PA. Perceptions of positive meaning and vulnerability following breast cancer: Predictors and outcomes among long-term breast cancer survivors. Ann Behav Med 2005;29:23645. Doi: 10.1207/s15324796abm2903_10

14. Mols F, Vingerhoets AJJM, Coebergh JW, Van de Poll-Franse LV. Quality of life among long-term breast cancer survivors: A systematic review. Eur J Cancer 2005;41:26139. Doi: 10.1016/j.ejca.2005.05.017

15. Hodgkinson K, Butow P, Hunt GE, Pendlebury S, Hobbs KM, Wain G. Breast cancer survivors' supportive care needs 2-10 years after diagnosis. Support Care Cancer 2007;15:515-23. Doi: 10.1007/s00520006-0170-2 
16. Rosedale M, Krainovich-Miller B. Confronting the unexpected more than a year following breast cancer treatment: A secondary analysis of phenomenological data. Ponencia presentada en el 10th World Congress of Psycho-Oncology, Madrid, junio 2008.

17. Given C, Given B, Rahbar M, Jeon S, McCorkle R, Cimprich B, et al. Effect of a cognitive behavioural intervention on reducing symptom severity during chemotherapy. J Clin Oncol 2004;22:507-16. Doi: 10.1200/JCO.2004.01.241

18. Lengacher CA, Johnson-Mallard V, PostWhite J, Moscoso MS, Jacobsen PB, Klein TW, et al. Randomized controlled trial of mindfulness-based stress reduction (MBSR) for survivors of breast cancer. Psychooncology 2009;18:1261-72. Doi: 10.1002/ pon.1529

19. Rehse B, Pukrop R. Effects of psychosocial interventions on quality of life in adult cancer patients: meta analysis of 37 published controlled outcome studies. Patient Edu Couns 2003;50:179-86.

20. Smith SK, Hemdon JE, Lyerly HK, Coan A, Wheeler JL, Staley T, et al. Correlates of quality of life-related outcomes in breast cancer patients participating in the Pathfinders pilot study. Psychooncology 2011;20:559-64. Doi: 10.1002/pon. 1770

21. Bellver A. Eficacia de la terapia grupal en la calidad de vida y el estado emocional en mujeres con cáncer de mama. Psicooncología 2007;4:133-142.

22. Die-Trill M. Tratamiento psicológico grupal para mujeres con cáncer de mama: una aproximación clínica. Psicooncología 2007;4(2-3):405-16.

23. García Lumbreras S, Blasco T. Características de la sexualidad en mujeres con cáncer de mama. Psicooncología 2008;5:15570.

24. Ochoa C, Sumalla EC, Maté J, Castejón V, Rodríguez A, Blanco I, et al. Psicoterapia positive grupal en cáncer. Hacia una atención psicosocial integral del superviviente de cáncer. Psicooncología 2010;7:7-34.

25. Kissane DW, Bloch S, Smith GC, Miach P, Clarke DM, Ikin J, et al. Cognitiveexistential group psychotherapy for women with primary breast cancer: a randomised controlled trial. Psychooncology 2003;12:532-46. Doi: 10.1002/pon.683

26. Michalec B. Exploring the multidimensional benefits of breast cancer support groups. J Psychosoc Oncol 2005;23:15979. Doi: 10.1300/J077v23n02_10

27. Spira JL, Reed M. Group psychotherapy for women with breast cancer. Washington: American Psychological Association, 2003. Doi:10.1037/10461-000

28. Berglund G, Bolund C, Gustafsson U, Sjödén P. A randomized study of a rehabilitation program for cancer patients: The "starting again" group. Psychooncology 1994;3:109-20. Doi: 10.1002/ pon.2960030205

29. Cella DF, Sarafian B, Snider PR, Yellen SB, Winocour P. Evaluation of a communitybased cancer support group. Psychooncology 1993;2:123-32. Doi: 10.1002/ pon.2960020205

30. Cunningham AJ, Lockwood GA, Edmonds CVI. Which cancer patients benefit most from a brief group coping skills program? Int J Psych Med 1993;23:383-98. Doi: 10.2190/EQ7N-2UFR-EBHJ-QW4P

31. Targ EF, Levine EG. The efficacy of a mindbody-spirit group for women with breast cancer: a randomized controlled trial. Gen Hosp Psych 2002;24:238-48.

32. Font A. Valoración de la calidad de vida en pacientes de cáncer. Tesis doctoral. Universidad Autónoma de Barcelona. BeIlaterra: Barcelona, 1988.

33. Font A. Cáncer y calidad de vida. Anu Psicol 1994;61:41-50.

34. Font A, Bayés R. Desarrollo de un instrumento para la medida de la calidad de vida en enfermedades crónicas. En: M. Forns M, Angera MT, compiladores. Aportaciones Recientes a la Evaluación 
Psicológica. Barcelona: Universitas-53, 1993.p.175-95.

35. Delgado-Sanz MC, García-Mendizábal MJ, Pollán M, Forjaz MJ, López-Abente G, Aragonés $\mathrm{N}$, et al. Health-related quality of life in Spanish breast cancer patients: a systematic review. Health Qual Life Outcomes 2011;9:1-10. Doi: 10.1186/14777525-9-3.

36. Padierna C. Medida de la calidad de vida en enfermos oncológicos terminales con el QL-CA-Afex. Proyecto de investigación. Universidad de Oviedo. Oviedo, 2000.

37. Espantoso R, Fernández C, Padierna C, Amigo I, Villoria E, Gracia JM et al. Calidad de vida en pacientes oncológicos un año después de finalizado el tratamiento. Psicooncología 2007;4:43-57.

38. Font A, Rodríguez E. Eficacia de la terapia de grupo en cáncer de mama: variaciones en la calidad de vida. Psicooncología 2004;1(1):67-86.

39. León C, Gich I, Barthe E, Rovirosa A, Farrús $B$, Casas $F$, et al. A randomized trial of the effect of training in relaxation and guided imagery techniques in improving psychological and quality-of-life indices for gynecologic and breast brachytherapy patients. Psychooncology 2007;16:971-9. Doi: 10.1002/pon.1171

40. Padierna C, Fernández C, Amigo I, Gracia JM, Fernández R, Peláez I, et al. Estudio longitudinal de los parámetros de calidad de vida en pacientes oncológicos. Psicooncología 2004;1(2-3):191-204.

41. Padierna C, Fernández C, González A. Calidad de vida en pacientes oncológicos terminales, medida con el QL-CA-AFEX. Psicothema 2002;14:1-8.

42. Banthia R, Malcarne VL, Ko CM, Varni JW, Sadler GR. Fatigued breast cancer survivors: the role of sleep quality, depressed mood, stage and age. Psychol Health 2009;24:965-80. Doi: 10.1080/08870440802110831

43. Deshields T, Tibbs T, Fan M, Bayer L, Taylor $M$, Fisher E. Ending treatment: the course of emotional adjustment and quality of life among breast cancer survivors immediately following radiation therapy. Support Care Cancer 2005;13:1018-26. Doi: 10.1007/s00520-005-0801-z

44. Härtl K, Schennanch R, Müller M, Engel J, Reinecker $\mathrm{H}$, Sommer $\mathrm{H}$, et al. Quality of life, anxiety and oncological factors: A follow-up study of breast cancer patients. Psychosomatics 2010; 51:112-23.

45. Schou I, Ekeberg O, Ruland CM, Sandvik L, Karesen R. Pessimism as a predictor of emotional morbidity one year following breast cancer surgery. Psychooncology 2003; 13:309-20. Doi: 10.1002/pon.747

46. Mehnert A, Koch U. Psychological comorbidity and health-related quality of life and its association with awareness, utilization and need for psychological support in a cancer register-based sample of long-term breast cancer survivors. J Psychosom Res 2008;64:383-91.

47. Schoen EG, Nicholas DR. Existential meaning through illness: the case of patients with breast cancer. J Psychosoc Oncol 2004; 22:61-76. Doi: 10.1300/ J077v22n04_04

48. Tartaro J, Roberts J, Nosarti C, Crayford T, Luecken L, David A. Who benefits? Distress, adjustment and benefit-finding among breast cancer survivors. J Psychosoc Oncol 2005;23:45-64. Doi: 10.1300/ J077v23n02_04

49. Dolbeault $S$, Cayrou S, Brédart A, Viala AL, Desclaux B, Saltel $P$, et al. The effectiveness of a psycho-educational group after earlystage breast cancer treatment: results of a randomized French study. Psychooncology 2009;18:647-56. Doi: 10.1002/pon.1440

50. Grassi L, Sabato S, Rossi E, Marmai L, Biancosino B. Effects of supportive-expressive group therapy in breast cancer patients with affective disorders: A pilot study. Psychother Psychosom 2010;79:3947. Doi: $10.1159 / 000259416$

51. Jones JM, Cheng $T$, Jackman $M$, Walton T, Haines S, Rodin G, et al. Getting back 
on track: Evaluation of a brief group psychoeducation intervention for women completing primary treatment for breast cancer. Psychooncology 2013;22:117-24. Doi: 10.1002/pon.2060

52. Manos D, Sebastián J, Mateos N, Bueno MJ. Results of a multi-componential psychosocial intervention programme for women with early-stage breast cancer in Spain:
Quality of life and mental adjustment. Eur J Cancer Care 2009;18:295-305. DOI: 10.1111/j.1365-2354.2008.00978.x 53. Sherman KA, Heard G, Cavanagh KL. Psychological effects and mediators of a group multi-component program for breast cancer survivors. J Behav Med 2010;33:378-91. Doi: 10.1007/s10865010-9265-9 
